\title{
L'Internat Scolaire: limites et paradoxes d'une institution totale
}

\author{
Michèle Guigue \\ Audrey Boulin" \\ 'Université Lille 3, Lille - France \\ "Université Paris-Est Créteil, Paris - France
}

RÉSUMÉ - L'Internat Scolaire: limites et paradoxes d'une institution totale. L'internat scolaire est une modalité de scolarisation qui offre hébergement et repas. Le temps dans un établissement scolaire est donc amplifié et concerne ce qui est habituellement distinct: l'entretien de la vie, le travail et les loisirs. Cette caractéristique en fait ce que Goffman nomme une institution totale. L'ampleur temporelle de cette emprise et la vie collective intense qui s'ensuit développent, d'une part, des relations personnelles et émotionnelles fortes, d'autre part, des arrangements et des pratiques cachées qui limitent la prégnance de cette institution.

Mots-clés: Internat Scolaire. Professionnels de l'École. Normes. Personnalisation. Institution Totale.

RESUMO - O Internato Escolar: limites e paradoxos de uma instituição total. O internato escolar é uma modalidade de escolarização que oferece alojamento e refeições. Dentro desse tipo de estabelecimento escolar, o tempo se amplia ao englobar o que é normalmente separado: a sobrevivência, o trabalho e os lazeres. Essa característica faz com que Goffman o considere como uma instituição total. A extensão temporal dessa influência e a intensa vida coletiva que ela acarreta permitem o desenvolvimento de relações pessoais e emocionais fortes por um lado, e por outro, certas disposições e práticas ocultas que limitam a ascendência dessa instituição.

Palavras-chave: Internato Escolar. Profissionais da Escola. Normas. Personalização. Instituição Total.

Educação \& Realidade, Porto Alegre, v. 41, n. 4, p. 985-1002, oct./déc. 2016. 985 http://dx.doi.org/10.1590/2175-623661105 
L'internat scolaire est une modalité de scolarisation qui permet aux élèves de bénéficier d'un hébergement et de services de restauration, aujourd'hui du lundi matin au vendredi soir. Le temps dans un établissement scolaire est donc amplifié. Peut-on considérer que cette modalité de scolarisation conduit à plus d'emprise de la part d'une institution soutenue par une forte légitimité?

Une multiplicité d'institutions éducatives contribue, simultanément et successivement, à la prise en charge des enfants et des jeunes. Leurs champs d'action sont très variés: instruction, sport, activités artistiques ou culturelles... Leurs objectifs sont hétérogènes: de la garde à l'éducation, de l'apprentissage à la distraction, de la prévention au soin, de l'initiation à la compétition. Orientées par des valeurs et des missions spécifiques, ces institutions ont souvent en commun une organisation structurée des pratiques, celles des professionnels comme celles des usagers. Cette socialisation plurielle et fragmentée place les individus face à des possibles ouverts, incertains, souvent embrouillés.

\section{L' Internat, d'Hier à Aujourd'hui}

L'internat scolaire a une longue histoire, il s'inscrit dans des contextes sociaux et historiques qui ont considérablement évolué. Pendant la Révolution Française, les questions d'éducation et de scolarisation ont été longuement débattues (Julia, 1981). L'internat a été envisagé clairement comme une stratégie, tout d'abord, de lutte contre les particularismes et les attachements locaux, sociaux et familiaux, mais aussi de promotion de justice sociale. Pour cela Le Pelletier de Saint Fargeau, en 1793, a conçu un projet qui prévoyait d'enlever les enfants de 5 à 12 ans à leur famille pour les élever dans des Maisons d'éducation communes. L'éloignement de la famille était considéré comme une condition nécessaire au bon développement des potentiels de l'enfant au service de son pays. "Les enfants appartiennent à la République avant d'appartenir à leurs parents" déclare Danton. Dans la tourmente révolutionnaire, aucun des projets débattus ne fut réalisé et leur caractère radical apparaît désormais comme utopique. Pourtant avaient émergé des préoccupations qui demeurent vivaces aujourd'hui: la lutte contre les inégalités, la justice sociale, le partage du travail éducatif entre la famille et l'école.

$\mathrm{Au}$ XIXe siècle, l'internat était un moyen nécessaire pour suivre une scolarité avancée. Les établissements secondaires étaient peu nombreux et s'adressaient à une minorité. Le " pensionnat ", comme on le disait alors, évoquait des établissements aux longs dortoirs, au confort rudimentaire et à la discipline de fer. Il était associé aux images négatives de prison, de couvent, ou de caserne. Les recherches en histoire de l'éducation croisent souvent l'internat (Prost, 1968; Mayeur, 2004), par exemple en s'attachant à la scolarisation des filles (Rogers, 2007) ou à celle des indigènes (Salaün, 2005). L'internat est aperçu et décrit

986 Educação \& Realidade, Porto Alegre, v. 41, n. 4, p. 985-1002, oct./déc. 2016. 
parce qu'il était un élément contextuel important. Cette logique instrumentale décroît, dans les années 1970, avec la multiplication des établissements secondaires, seul le choix de filières rares (sports-études...) conduit à ce recours.

Ainsi après être tombé en désuétude, l'internat scolaire a réapparu dans les discours politiques et un Plan de relance de l'internat scolaire public est lancé en 2000. Ce plan s'appuie sur des orientations fortes: éloigner certains jeunes de leur famille et de leur quartier défavorisé pour lutter contre les inégalités en leur offrant de meilleures conditions d'études et pour les protéger de la délinquance, favoriser la mixité sociale. L'internat est un moyen envisagé pour soutenir un projet éducatif, scolaire, social et culturel, car il propose, quantitativement, plus d'école et, simultanément, plus de distance par rapport au milieu des élèves. En 2008, l'internat figure dans le cadre de la politique de la ville sur le thème Espoir Banlieue, il s'agit de revitaliser les internats existants, puis, en 2010, sont créés les Internats d'excellence. Leurs caractéristiques principales étaient-le recrutement sur profil des professionnels comme des élèves, - l'ouverture de financements favorables notamment pour l'usage de la plage horaire $17 \mathrm{~h}-21 \mathrm{~h}$, - des classes réduites à 20 élèves. Cette catégorie administrative a été supprimée en 2013, à la suite d'un changement de majorité politique. Malgré son existence éphémère, elle a été exemplaire d'une nouvelle prise en compte des questions soulevées par l'échec du système scolaire à diminuer les inégalités: elle conjuguait des innovations organisationnelles et un projet pédagogique ambitieux pilotés d'en haut. Désormais, il s'agit d'impulser L'internat de la réussite pour tous. Sans tous les moyens des internats d'excellence, l'internat est conçu comme "un espace libérateur" et un outil de réduction des inégalités ${ }^{1}$.

\section{L'Internat Scolaire, État de la Question}

Dans le cadre de ce renouveau, l'internat scolaire a fait l'objet d'études et de rapports pour accompagner le plan de relance (France, 2000; France, 2002). Ces textes ne sont pas à proprement parler des écrits de recherche, ils ont été rédigés par des inspecteurs généraux qui visitent les établissements scolaires, mais qui, du fait de leur statut, sont au cœur des logiques organisationnelles. Ils décrivent un panel de situations particulières grâce auxquelles ils repèrent un certain nombre de caractéristiques clés: la nomination des professionnels, leur formation, l'impact de ce cadre sur le pilotage de ce type d'établissement. Ils formulent des recommandations parmi lesquelles: développer de véritables projets d'internat, prévoir des temps forts pour la deuxième journée des internes, formuler des règles de façon moins caporaliste et plus participatives, recruter un encadrement suffisant, qualifié et motivé. Le poids du milieu de vie créé par l'internat est ainsi abordé, son évolution est envisagé avec volontarisme. Il s'agira de surmonter des freins institutionnels et financiers. 
Durant cette période le sociologue Dominique Glasman participe aux travaux de réflexion du ministère et conduit une recherche centrée sur l'usage que les élèves et leurs familles font de l'internat (Glasman, 2012). Elle se situe dans la continuité de ses travaux sur le recours des parents à des dispositifs marchands pour soutenir la scolarité de leurs enfants. Dans sa présentation de l'offre d'internat, il souligne les différences entre, d'une part, les établissements privés qui développent des projets éducatifs liés à cet accueil, soutien et activités diverses et, d'autre part, les établissements publics qui s'en tiennent, souvent, à de simples services, c'est-à-dire le gîte et le couvert. Une thèse a été soutenue sur l'internat d'excellence par Audrey Boulin. Elle y analyse ce dispositif de façon monographique, sur un terrain, sous l'angle de l'expérience adolescente (Boulin, 2014). Elle y montre que les jeunes modifient leur sociabilité, leurs loisirs et leur travail scolaire, mais pas toujours comme cela avait été espéré. La création de nouveaux liens mêle quête de plaisir et considérations plus stratégiques de rentabilité scolaire en choisissant, parmi les camarades, des partenaires de travail. L'enjeu de mixité sociale est en partie transformé par le fait qu'une majorité des internes de cet établissement est adepte de la culture de rue.

Avec la création des Internats d'excellence, des recherches ont été commanditées pour évaluer leur efficacité (Rayou; Glasman, 2012; Behaghel; De Chaisemartin; Charpentier; Gurgand, 2013). L'appréciation de leur impact sur l'amélioration des performances scolaires des élèves est plutôt positive, mais nuancée. Toutefois la brièveté de leur existence fragilise tout résultat quel qu'il soit.

\section{Problématique}

L'institution scolaire impose une mise en forme des apprentissages, dans un espace à l'écart et protégé, selon une temporalité précisément planifiée, dans un cadre collectif où les règles sont impersonnelles, autrement dit valent pour tous de façon indifférenciée. Ses normes dominent les autres démarches éducatives par leur caractère obligatoire tout au long de l'enfance et de la jeunesse, par leur impact sur l'itinéraire personnel, social et professionnel.

Avec l'internat, l'école dispose quotidiennement de temps qui sont habituellement gérés librement par les jeunes et leurs parents, à l'abri du regard de l'école. C'est un type d'établissement dans lequel se déroulent des activités qui appartiennent aux trois champs de la vie ordinaire, celui de l'entretien de la vie (repos et sommeil, alimentation, hygiène), celui du travail (cours et études surveillées) et celui de la détente et du divertissement. Cette caractéristique correspond aux critères que Goffman retient pour construire le concept d' institution totale. Il les a défini précisément par comparaison avec les conditions de vie contemporaine: 
C'est une caractéristique fondamentale des sociétés modernes que l'individu dorme, se distraie et travaille en des endroits différents, avec des partenaires différents, sans que cette diversité d'appartenance relève d'un plan d'ensemble. Les institutions totales, au contraire, brisent les frontières qui séparent ordinairement ces trois champs d'activité; c'est même là une de leurs caractéristiques essentielles (Goffman, 1968, p. 47).

Ce terme total qualifie donc une emprise transversale sur des activités d'ordre différents car les membres sont tenus à résidence. Cette emprise tient à ce que toutes les activités sont

[...] réglées selon un programme strict en sorte que toute tâche s'enchaîne avec la suivante [...] conformément à un plan imposé d'en haut par un système explicite de règlements dont l'application est assurée par une équipe administrative (Goffman, 1968, p. 48).

Cependant, malgré cette emprise, malgré une délimitation par quatre murs, ces organisations sont "[...] riches en activités clandestines, car les chances de succès total de toute programmation diminue au fur et à mesure que s'allonge la durée du temps programmé" (Goffman, 1968, p. 258). Les travaux de l'historienne Agnès Thiercé (1999) qui décrit les révoltes lycéennes violentes du XIXe siècle, l'attestent. Malgré l'intensité de la surveillance, la rigueur des règles de vie et des sanctions de cette époque, les jeunes restaient en prise sur les soubresauts politiques du moment. L'école n'est pas un sanctuaire isolé, à l'abri du monde. L'internat scolaire assure une mise à distance et une clôture tout à fait relative.

Goffman souligne que, même dans un cadre contraignant et omniprésent, il existe une vie clandestine (underlife) et des arrangements (conways) entre les professionnels et les usagers, arrangements qui fissurent la rigidité de ses normes et modèrent son emprise. Cette reconnaissance est liée à sa conception de la participation sociale: celle-ci n'est pas monolithique. Les acteurs, même motivés et volontaires, sont animés par des tensions entre le pénible et l'agréable, entre l'adhésion et la résistance, autrement dit par de l'ambivalence et de l'ambiguïté. Éclairées par cette perspective, nous nous demanderons comment l'internat scolaire fonctionne, aujourd'hui, au quotidien? Dans quelle mesure l'unité de lieu pour entretenir sa force de vie, travailler et se distraire se fait-elle au profit du travail scolaire? Comment les différentes catégories d'acteurs s'approprient-elles ce cadre institutionnel?

\section{Méthodologie de Collecte et d'Analyse}

Des données ont été collectées dans deux internats, Les érables durant 2 années scolaires de 2010 à 2012 et Les platanes durant 4 années de 2011 à $2015^{2}$. Ce sont de petits établissements qui comprennent, selon 
les années, de moins de 100 à 180 élèves. Au cours de cette recherche qualitative, nous avons conduit une centaine d'entretiens auprès de professionnels (cadres, enseignants, assistants d'éducation, personnels d'entretien), de parents et d'élèves. De plus, nous avons observé, sur le mode ethnographique (Guigue, 2012; 2014), tout au long de journées entières, la vie de ces établissements: réunions de toutes sortes, situations du quotidien dans les couloirs, les bureaux, les cours de récréation, les restaurants scolaires, et ponctuellement dans des salles de classe. Nous avons relu et interprété ces données en nous attachant, dans le cadre de ces deux établissements, à ce qui tenait à la vie en internat.

Ce qui a été dit dans les entretiens et ce qui a été vu lors des observations a été entrecroisé afin d'approcher les pratiques qui émaillent le quotidien: choix, modalités d'organisation, arrangements, vie clandestine. Cet entrecroisement permet de contourner les discours conformistes sur les intentions et les rôles attendus, ou encore de repérer d'éventuelles vantardises. Des arrangements et des pratiques cachées ont été entrevues lors de nos déambulations ou dévoilées à l'occasion d'incidents divers. Elles nous ont été racontées par des élèves confiants grâce à la longue durée de notre présence sur les terrains et satisfaits d'avoir l'occasion de montrer leurs astuces et leurs prouesses. Elles nous ont aussi été racontées par des assistants d'éducation qu'elles ont choqués et qui s'interrogeaient sur la manière de réagir.

Nous allons donc présenter, en quatre parties, l'internat scolaire comme institution totale, en pointant ses paradoxes ou ses limites. Tout d'abord, l'internat est un possible choisi qui n'est pas imposé par l'institution scolaire. Puis nous montrerons que l'internat est, incontestablement, plus d'école. Mais, paradoxalement, ce sera la $3^{\mathrm{e}}$ partie, ce plus d'école implique une vie collective intense qui contribue à fragiliser l'ordre scolaire traditionnel. Enfin, en 4e partie, nous présenterons les arrangements et les pratiques cachées qui limitent l'emprise de ce type d'établissement.

\section{L'Internat, un Choix Familial pour Réussir}

L'internat est une décision familiale qui associe parents et enfants et ne se prend pas à la légère. Cette inscription tient, en grande partie, à un objectif familial prioritaire de réussite sociale et professionnelle pour laquelle l'école est considérée comme incontournable. Pour de nombreux parents, l'internat permet d'échapper à l'établissement du secteur, dont la réputation, la ségrégation sociale, le climat ou les performances sont soupçonnés et dénigrés. La mère de Latifa (2nde) est claire: "C'était hors de question qu'elle aille au lycée de secteur. Il a une très mauvaise réputation et tout ça [...]". Ils font donc des démarches, acte de candidature et constitution d'un dossier, pour piloter eux-mêmes la scolarité de leur enfant, à distance de la mécanique administrative de l'école qui procède à des inscriptions automatiques 
en fonction de l'adresse du domicile. Mais ce mode de scolarisation implique aussi l'acceptation du jeune. C'est manifeste quand, dès le jour de la rentrée, Arthur lance des injures virulentes et publiques à l'égard de l'équipe de direction pour être exclu. Les motifs des adolescents pour accepter ce mode de scolarité portent majoritairement sur le domaine scolaire: "Je pensais qu'en internat, je serais plus cadré et je pourrais mieux faire mes devoirs " dit Adrian ( $3^{\text {ème)}}$. D'autres résistent, mais finissent par accepter: Cédric (5ème): "J'ai fait une lettre de motivation, ma mère me dictait les mots. Je voulais leur envoyer un truc du genre: en fait j'ai pas envie d'y aller parce que c'est nul. Au début même, j'ai pensé à me barrer, mais maintenant on s'y habitue". Ces jeunes ont intériorisé, bon gré mal gré, le désir de leur parents et les normes scolaires au nom de l'avenir.

En une période où l'enfant occupe une place centrale dans la famille et est porté par un projet éducatif ambitieux, le choix de l'internat qui implique de l'éloignement peut étonner. D'ailleurs, l'épreuve renouvelée de la séparation est manifeste, pour les uns et les autres, quand les soirées commencent. Parents et enfants adhèrent à l'école, ils ont intériorisé ses valeurs. Mais ils ne considèrent pas l'école comme un bloc monolithique: ils ne font pas confiance à ses procédures administratives ordinaires. Ils s'informent, font des démarches particulières et sont prêts à ces sacrifices pour se donner de meilleures chances: accéder à un établissement de qualité, s'éloigner des problèmes du quartier.

Dans cette stratégie l'école n'est pas prise en compte pour ellemême mais comme un moyen au service de finalités. Les parents analysent leur situation et celle de leur enfant en prenant en compte d'autres contraintes lourdes, celles du quartier et de leur appartenance sociale ou ethnique... Lécole peut être écrasante, mais la relation à l'école n'est pas pensée de façon isolée (Guigue, 2013), elle peut être un recours pour contrer, atténuer ou contourner d'autres emprises. Ils choisissent, comme Bourdieu (1987) le souligne, en fonction du jeu dont ils disposent, les atouts ou les mauvaises cartes.

Ainsi, les parents invoquent aussi la sphère familiale: leurs conditions de logement, les problèmes d'encadrement liés à leurs contraintes professionnelles, des relations conflictuelles entre eux à l'occasion d'un divorce, ou encore avec leur enfant ou dans la fratrie. "Je travaille énormément donc le soir je rentrais tard. Elle était confiée à la responsabilité de ses grands-parents mais, bon, je la retrouvais souvent chez ses copines, et les devoirs sont pas faits quoi!" dit la mère de Muriel (4ème). L'internat est perçu comme un cadre de travail solide propice à la réussite. Des mères choisissent un internat pour soustraire leurs filles à la

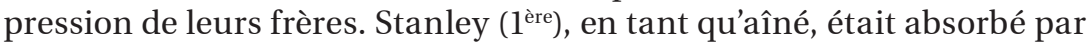
des tâches familiales: "Il fallait que j'aille chercher les petits à la primaire. Après il fallait que je les aide eux. Déjà, du coup, ça faisait pas mal de temps perdu". Il a intégré l'internat pour avoir du temps pour sa scolarité. 
La rhétorique des mauvaises fréquentations est fréquente. Des parents se mobilisent pour éloigner leur enfant d'un quartier inquiétant ou d'un entourage exerçant une influence néfaste. Le père de Fahima (4ème), gardien de nuit résidant dans une zone urbaine dite sensible, explique: "Elle avait vraiment des difficultés comportementales, c'était pas des difficultés psychologiquement, elle se bagarrait avec des copines. Ça a commencé depuis l'école primaire, le changement est arrivé lorsqu'elle a été séparée d'un groupe de filles avec qui elle avançait au fil des années". Les élèves de quartiers défavorisés reprennent souvent à leur compte cet argument, comme David (3ème): "En fait, avec mes potes, on faisait quelques bêtises, mais tranquille. On apprenait plus les cours, j'ai commencé à sécher avec eux. On restait dans le hall de mon immeuble et, quelques fois, on allait voir les grands et on faisait des trucs pour eux, voler des vélos". De même Océane (3ème) raconte: "En 4 ème, je me battais en bande et tout. On m'a dit que si j'étais restée làbas j'allais devenir une délinquante. À l'école, je m'engueulais toujours avec mes profs. On se baladait, on se réunissait pour frapper des gens d'autres villes qui venaient dans notre quartier. Ils avaient pas le droit".

L'emprise de l'école est parfois pénible, mais, pour parents et élèves, elle n'est pas isolable d'autres emprises, celle de la famille, du quartier ou de fréquentations déviantes. Elles aussi peuvent être pénibles mais, surtout, elles sont clairement identifiées comme problématiques pour l'avenir.

\section{L'Internat, c'est plus d'École}

L'inscription en internat est perçue comme l'accès à un cadre protecteur. Certes, cela ne va pas sans contrepartie: l'internat scolaire limite la liberté par l'extension d'un cadre scolaire rigoureux qui se diffuse au delà des missions centrales de l'école, à l'ensemble du quotidien. Ces contreparties sont même souhaitées explicitement.

\section{Un cadre spatio-temporel rigide}

Le choix de l'internat est soutenu par le souhait d'études aux horaires encadrées pour être poussé à travailler et aidé pour acquérir des méthodes de travail. Aux heures de cours succèdent donc des études surveillées, parfois des ateliers, des accompagnements personnalisés divers. Ce qui est habituellement dissocié, le travail à l'école et les devoirs à la maison, s'enchaîne sans que les lieux soient vraiment différenciés (France, 2002). En effet, les locaux de l'internat ne sont ouverts et accessibles qu'après le repas du soir quand vient, pour beaucoup, le moment de se détendre, de téléphoner aux parents et aux amis.

Les élèves, comme les pensionnaires de l'hôpital Sainte Catherine observés par Goffman, sont à la recherche de lieux confortables, du moins, où ils puissent s'affaler comme ils le feraient s'ils rentraient chez 
eux. À l'internat, ils doivent toujours avoir une position correcte, le corps redressé (Vigarello, 2004). Où et quand est-il possible ou acceptable de se détendre, intellectuellement, corporellement? Sur quelques fauteuils dans le centre de documentation. Les abords, les bâtiments, les couloirs et leurs nombreuses portes offrent néanmoins des opportunités. Des élèves qui découvrent une porte non verrouillée s'installent tranquillement dans un local technique pour discuter. D'autres s'esquivent dans le parc, dans le gymnase mitoyen... L'important est de repérer des horaires judicieux et de ne pas se faire prendre. Fanny (lère) ne semble pas s'esquiver fréquemment, mais de se rendre compte "qu'en fait le mercredi après-midi, on pouvait sortir et rentrer à $20 \mathrm{~h}$ (Rires), donc ça allait mieux". Se soustraire à l'enveloppe spatiale et normative de l'établissement, ou s'imaginer le faire, est plaisant.

Les personnels portent une grande attention à l'organisation temporelle, en enchaînant des temps contraints. Les repas, les récréations sont minutées et, parfois, se chevauchent. Aujourd'hui comme hier, face à ce public adolescent, il y a la peur du vide comme le notait Agnés Thiercé décrivant les internats du XIXe siècle (Thiercé, 1999, p. 63). Certains élèves revendiquent du repos, des siestes (sic). Le temps libre des internes s'apparente parfois à celui des pensionnaires du passé: en 1872, le surmenage apparaissait dans une circulaire et était dénoncé (Mayeur, 2004). Le temps libéré de toute contrainte est fort limité dans l'emploi du temps, il se trouve souvent réduit à des interstices et il est absent de la réflexion des professionnels tout entiers mobilisés par l'organisation des apprentissages scolaires et culturels.

\section{Des Interférences entre Scolaire et Loisirs}

L'ampleur des horaires et la nécessité de les occuper conduisent à proposer des activités culturelles, sportives ou de loisirs, en fin de journée et les mercredi. Chaque internat a ses propres stratégies en fonction des ressources offertes par son environnement (montagne, mer, équipements culturels...) et des financements obtenus grâce au dépôt de projets. Selon les cas, ils font découvrir des loisirs culturels perçus comme légitimes, sorties au théâtre, au concert... ou bien des activités appréciés par les jeunes, arts martiaux, danse hip-hop, football féminin... Il est alors mal vu, voire impossible, de ne rien faire. Romain (lère) s'insurge: "J'ai été inscrit par force à l'activité culturelle". Élodie (2de) a été face à un dilemme: choisir entre le sport qui comprenait la natation, or elle a peur de l'eau et le théâtre que sa timidité lui faisait redouter.

À l'extérieur, ces activités seraient choisies et considérées comme des loisirs. Dans le cadre contraignant de l'internat, elles mériteraient, parfois, le qualificatif scolaire d'options qui désigne les variations possibles dans la programmation d'un cursus. Un paradoxe de cette prégnance de l'internat sur l'ensemble du quotidien tient à ce qu'elle ouvre sur des activités extra-scolaires dont les élèves ne bénéficieraient pas 
dans un établissement ordinaire. Mais simultanément, ces activités deviennent scolarisées et la frontière entre le travail et le divertissement se trouve brouillée.

Cependant l'impression d'emprise liée à ce brouillage n'affecte pas tous les élèves. Certains sont pris dans une convivialité chaleureuse: les championnes de boxe qui sont convoyées de compétitions en compétitions par leur professeur d'éducation physique et sportive, Maximilien qui est entraîné par les enseignants de lettres pour des concours de rhétorique... D'autres sont si focalisés sur leur volonté de réussite qu'ils s'accommodent de ces contraintes: les activités culturelles assurent leur développement personnel au profit de leur projet professionnel et le sport les maintient en bonne forme pour soutenir un rythme de travail intense. Ils endossent la stratégie et les normes de l'école en espérant qu'elle va leur ouvrir un bel avenir grâce auquel ils auront, plus tard, plus de liberté. L'école est une étape dans un itinéraire de vie, son emprise présente en devient supportable.

\section{Un Encadrement par des Professionnels de l'École}

Les personnels qui travaillent dans les internats, de l'équipe de direction aux assistants d'éducation, sont recrutés par l'institution scolaire selon des profils valant pour tous les établissements. Les assistants d'éducation qui veillent aux moments de la vie quotidienne, soirées et nuits, lever et douches.... ne sont jamais des éducateurs ou des animateurs formés. Ce sont de jeunes adultes qui commencent leur vie salariée par ces fonctions transitoires qui amorcent un chemin vers une professionnalisation stable s'ils passent des concours et les réussissent. L'équipe de direction, l'infirmière, sont logées sur place, ils assurent des astreintes: ils sont joignables à toute heure le soir et la nuit. Alertés, ils arrivent immédiatement et prennent en charge malaise, crise d'angoisse, perturbation diverses.

La proximité répétitive lors d'activités domestiques (repos et sommeil, alimentation, hygiène) suscite des relations intimes, à forte composante émotionnelle. De plus, les effectifs réduits rendent l'anonymat impossible. Les élèves sont des enfants dont des fragments de l'histoire et de la vie familiales sont connus. Marie, une assistante d'éducation s'interroge: est-il vraiment judicieux de surveiller des temps scolaires, puis des temps en internat? Pour les premiers, elle se dit rigide et n'hésite pas à crier, pour les seconds, elle est cool. Elle a l'impression qu'elle est face des contradictions.

Faire tout ou partie de son service en internat fait éclater les configurations des compétences attendues et le partage du travail (Hughes, 1971). Qui vérifie que tel ou tel va bien à son rendez-vous chez le psychiatre, le médiateur de justice... Qui est là pour rassurer? Quand convient-il d'appeler le cadre d'astreinte? Les professionnels se trouvent pris dans un ensemble de situations qui débordent largement ce qui 
relève du scolaire et des tâches pour lesquelles ils ont été formés. Les élèves confient leurs soucis scolaires ou familiaux. Louis est perturbé par le divorce très conflictuel de ses parents. Il sait que sa mère téléphone souvent en espérant se faire des alliés pour le jugement prochain. La conseillère d'éducation cherche à pacifier la situation et à rassurer Louis. L'internat ne le met pas totalement à l'abri, mais les professionnels s'attachent à limiter les interactions pénibles entre Louis et sa mère. Les professionnels entretiennent, involontairement, avec les jeunes des relations personnelles colorées par de l'affectivité, sympathie, bienveillance, compassion, mais aussi irritation.

\section{Une Vie Collective Intense}

La proximité à longueur de journée, les effectifs réduits créent un contexte relationnel où tous, jeunes et adultes, se connaissent et se côtoient, qu'ils le souhaitent ou non. Pour les élèves, vivre en internat fait éprouver le poids du regard des autres d'autant plus que les impératifs liés à la surveillance et à la sécurité supposent que, la plupart du temps, ils soient rassemblés.

\section{Tensions et Émotions}

Les premiers jours de séparation sont solitaires. Les amis sont loin et tisser de nouveaux liens est important, voire urgent. C'est le cas pour

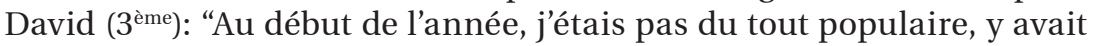
personne qui venait me voir. C'est deux semaines après la rentrée, la toute première bagarre qui m'a fait connaître. Et après mon prénom il a commencé à tourner dans tout l'internat". L'éloignement ne garantit pas le développement de manières de vivre ensemble différentes.

Les chambres sont rarement individuelles et les moments d'isolement sont souvent limités à des interstices. Des internes s'en plaignent: "J'en ai marre d'être tout le temps dans un bain de gens. J'aime bien être la première dans la chambre parce que, comme ça, j'ai deux trois minutes pour souffler un peu vraiment toute seule" Mathilde (5ème). La proximité peut devenir promiscuité. Certains mettent des écouteurs pour se créer une bulle. Mais il y a beaucoup d'ambivalence dans ce rapport au collectif: il permet de s'ouvrir, de "ne pas rester timide" dit Anaya, et ceux qui s'en vont regrettent de ne plus être entourés.

Le jugement des pairs trouve de multiples occasions pour s'exprimer. Le climat est souvent chargé d'émotions intenses qui peuvent passer rapidement de l'agréable au pénible. Dans ce monde mixte, les flirts sont nombreux, mais ils sont aussi éphémères. Marwan a été lâché par son amie. Lors de la fête de Noël, il avait déclaré son amour spectaculairement, à genoux devant sa belle en lui offrant une bague de valeur. Dès le retour des vacances, elle s'affiche avec morgue auprès d'un autre. Marwan, lui, a les deux mains dans des pansements dignes de gants 
de boxe. De tristesse et de rage, il a tambouriné un mur, il s'est cassé un doigt et se traîne la mine sombre. Sa mère est appelée pour venir le chercher et l'éloigner quelques jours de ce voisinage éprouvant. Nombreuses sont ces histoires, certaines sont des découvertes amoureuses empruntes de légèreté, d'autres sont marquées par des souffrances. Et il est impossible de s'éviter, d'échapper au regard de son remplaçant, ni à celui des autres. Les adultes compatissent et s'inquiètent.

Avoir des ami(e)s est important, c'est la garantie d'un soutien réciproque, mais ce peut être une source de malaise. Les deux grandes amies de Pauline n'ont pas repéré son absence. C'est un enseignant qui s'est inquiété pour cette fille fragile, où était-elle? Tous les professionnels se sont mobilisés pour explorer les moindres recoins, et ils ne manquent pas. Elle a été retrouvée recroquevillée, sous un lavabo, à l'étage des filles. Cela a pris du temps car les portes auraient dû être fermées, elle n'aurait pas dû pouvoir se réfugier là... Le pire avait été imaginé, c'était juste une alerte. Ses amies ont très affectées, comment avaient-elles pu ne s'apercevoir de rien?

Les remarques désobligeantes ne sont pas rares. Kévin jette à la figure de Nicolas Espèce d'homo!. Lors de son passage en conseil de discipline, il ne comprend pas et répète en boucle: "Mais puisque c'est vrail". Les rumeurs sont plus insidieuses, comment être sûr de qui en a eu l'initiative? Dénoncer, n'est-ce pas s'exclure du groupe? Céline raconte à cet interlocuteur extérieur qu'est l'enquêtrice: "Une fille a commencé à faire répandre la rumeur, qui est totalement fausse, que j'utilisais un sextoy. Voilà! Et puis elle commençait à en parler aux garçons. Et c'est totalement faux. Et même si c'était vrai, c'est pas son problème. Tellement ça m'a énervée, je voulais quitter l'internat, j'en avais marre!". Pourtant partir ne lui apparaît pas comme une bonne stratégie: quelles autres difficultés faudrait-il affronter?

Les professionnels sont déstabilisés par ces phénomènes de groupes, par l'impact émotionnel qu'ils ressentent face à ces situations délicates ou aux souffrances de ces adolescents. Ils s'interrogent sur la pertinence de leurs pratiques et n'ont personne à qui en parler.

\section{Des Arrangements plutôt que des Conflits}

L'internat crée une conjonction de proximité et de contraintes qui suscite des inquiétudes. Les professionnels, en poste pour la première fois en internat, craignent des débordements. Cette perspective leur semble d'autant plus grave que de nombreuses plages horaires ne sont pas cadrées par des cours. La première année, les règles sur la correction de l'habillement, la tenue à la cantine, l'usage du téléphone, la cigarette, le manque de respect... sont appliquées avec rigidité, les punitions sont sévères au point qu'il y a des exclusions. Au fil du temps, les uns et les autres apprennent à se connaître. La vie de ces petits établissements s'avère paisible. Les professionnels qui enseignent simultané- 
ment dans plusieurs établissements vantent l'internat, son calme, son cadre agréable, ils sont écoutés.

Le téléphone portable rend facile les communications avec l'extérieur, il a créé une zone floue. La régulation de son usage a évolué rapidement. Au début des années 2010, son encadrement était strict. En internat, ils étaient récupérés et distribués pour être disponibles une heure trente chaque jour, le soir entre le dîner et le coucher. Mais la collecte et la distribution quotidienne était une tâche lourde. Rapidement les surveillants ont repéré des ruses, de nombreux élèves possédaient plusieurs téléphones et remettaient celui dont ils ne se servaient pas. De plus, les parents considéraient que le téléphone était un accessoire indispensable, un lien essentiel avec leur enfant, leur assurant la capacité d'échanger et de réagir à tous moments. Les confiscations suscitaient des réclamations agressives de leur part et, parfois, ils accusaient les personnels d'avoir utilisé et vidé la carte SIM. Dès la rentrée 2012, les élèves conservent leur téléphone, les restrictions d'usage sont mentionnées dans le règlement intérieur. De fait, seul son usage en cours est vraiment sanctionné, s'il est repéré. La volonté de contrôle des téléphones portables n'a pas pu résister aux actions conjointes des élèves et de leurs parents. L'institution scolaire et les professionnels ont donc dû abandonner les différentes procédures mises en place au fil des années.

Cependant les domaines où il y a des arrangements entre les différents acteurs ne manquent pas. Le type d'arrangement le plus fréquemment repéré est l'intervention au cas par cas, en prenant en compte la situation du jeune. Quand Jordan s'emporte, néglige son travail, chaque année à la saison où sa mère s'est suicidée, tout le monde s'attache à laisser passer ce mauvais cap, même s'il y aurait motifs pour une exclusion. En dernière année de lycée, cela va mieux, les professionnels s'en félicitent. Ils avaient l'impression de bricoler, mais surtout de faire deux poids deux mesures. Leur principe de justice se trouvait questionné.

Une seconde modalité d'arrangement est la négociation. Souvent elle est individuelle, par exemple quand des élèves demandent à changer de chambre, ou quand, un par un, ils demandent à sortir d'étude sous différents prétextes: aller au Centre de documentation, récupérer un ouvrage dans son casier... Elle est aussi collective, dans les deux internats, l'horaire du coucher est contesté sans succès: "Ah nous on dort pas, c'est sûr, 22h, c'est trop tôt! Même quand on est fatigué. En plus, tu viens juste de rentrer dans ton lit, t'es encore en train de parler" Océane ( $3^{\text {ème)}}$. Aux Platanes, la direction a été accusée d'avoir conçu un emploi du temps plus favorable pour la classe de terminale scientifique, la plus cotée. À l'occasion d'une heure de permanence, les élèves des autres classes ont refusé de retourner en cours jusqu'à ce que leur emploi du temps soit aligné sur celui de la terminale scientifique: moins de trous dans l'emploi du temps, un départ de bonne heure le vendredi après midi. Ils ont obtenu gain de cause. Cette différence n'était pas volontaire, mais, de fait, elle était incontestable. 
Une troisième modalité d'arrangement est, ce que l'on peut appeler un accord tacite. Un aspect du règlement intérieur n'est pas respecté, mais tout le monde ferme les yeux. Ces infractions sont d'ordre divers: Les quantités offertes dans les repas, surtout le soir, ont été l'objet de récriminations. Ponctuellement, les élèves entreposaient des sandwichs dans leurs casiers et se les partageaient, au grand dam du professeur de biologie qui imaginait, en aparté, le risque d'une intoxication alimentaire. - Le soir les lumières restent allumées, les bavardages discrets ne sont pas sanctionnés. - Le manque de respect des heures d'étude est plus visible, mais les assistants d'éducation font juste des commentaires anodins. - De même, l'injonction vestimentaire décidée par certains internats: les joggings doivent être portés exclusivement pour les activités sportives, n'est pas suivie. Or l'habillement est un enjeu symbolique important.

Les vêtements conduisent directement au principe de laïcité et à son interprétation en internat. En France, le voile et les signes religieux visibles sont interdits. Des développements précisent en tous lieux et en toute occasion. La radicalité de ces expressions est sans ambiguïté, une distinction institutionnelle entre espaces publics (classes, couloirs...) et espaces privés (chambre) est implicitement refusée. Mais, pratiquement, qu'en est-il avec des élèves qui portent le voile dans leur chambre? Cette question n'est pas de l'ordre de l'hypothèse: ici ou là, des filles mettent un voile, notamment pour la prière. Elles se retrouvent dans leur chambre avec d'autres qui portent ou non un voile. Elles sont observables sur le seuil. Ce sont des détails, mais ce qui caractérise le domaine normatif, comme ce qui compose la vie en commun, ses agacements ou sa quiétude, n'est-ce pas un foisonnement de détails? Les adultes semblent ne rien voir. Dans une perspective éducative, une question centrale se pose: quel message envoie-t-on aux élèves, à celles qui portent un voile, mais aussi à tous ceux et celles qui les côtoient? Une valeur fondamentale du service public d'éducation est édictée dans des règles écrites sans nuance mais leur application est négligée. Estce le résultat réfléchi d'une distinction pratique entre espace public et espace privé? Un point est sûr: c'est un arrangement de fait, sans verbalisation.

\section{Des Pratiques Cachées}

Il y a aussi des pratiques prohibées, considérées comme trop problématiques pour faire l'objet de tentative d'arrangement. Elles s'opèrent dans les coulisses, soigneusement cachées, elles sont difficilement observables. En revanche, elles reposent sur une observation attentive pour repérer des interstices ou des failles dans l'organisation.

En début d'année, ces pratiques ont exploré la possibilité de neutraliser les détecteurs de fumée par de la mousse à raser ou des spray divers. Il est plus grave de s'esquiver jusqu'à franchir les murs d'enceinte 
pour fumer, s'amuser, rencontrer des amis, voire acheter du cannabis... Mais ce n'est pas impossible et jouer au chat et à la souris fait éprouver des sensations fortes, éloignées de la monotonie du quotidien. Paul (2nde) explique: "On était censés sortir que le mercredi après-midi et comme j'avais pas cours le mardi après-midi, je sortais aussi. J'essayais de pas croiser les surveillants, et voilà". Ray (T), quant à lui raconte, des sorties nocturnes lors de sa seconde année: "L'organisation était ramollie un petit peu, cela dépend aussi du surveillant et de l'heure à laquelle il pointe la présence dans les chambres. Des fois, quand je vois qu'il est encore tôt, 22h30, je prends le risque de... m'évader (sourire). Personne n'est au courant, à part Tiago qui me couvre (rire)". Il lui est arrivé d'avoir été pris. Quelle a été la punition? L'entretien se poursuit sans que cet aspect soit évoqué, c'est systématiquement le cas dans les récits d'infraction. L'excitation et le plaisir de déjouer l'emprise institutionnelle se suffisent à elles-même et font négliger la punition. On ne découvre les sanctions que par l'observation d'élèves convoqués par l'équipe de direction ou par la tenue de réunions ou de conseils de discipline.

Les pratiques cachées sont parfois enchâssées dans des cadres autorisés, elles passent ainsi d'autant plus facilement inaperçues. Jules est sorti avec Ana, sur une plage horaire autorisée, pour acheter du cannabis. Le fume-t-il uniquement chez lui, en fin de semaine? Il a été dans un quartier très mal famé, il s'est fait tabasser. Sa camarade a essayé de le défendre, elle a pris des photos et a appelé l'internat. Jules a des traces au visage et sur le corps. L'infirmière espère que la peur et le malaise qui l'habitent encore va le décider à s'arrêter. Quant à Ana elle a été félicitée pour sa présence d'esprit, mais aussi encouragée à la prudence pour sa propre sécurité.

À l'occasion d'une animation conduite par des intervenants extérieurs, les professionnels découvrent, interloqués, qu'une classe a un climat malsain très pénible. Une élève entretient systématiquement des tensions et du harcèlement. Personne ne s'en était aperçu et personne n'avait rien laissé filtré.

La vie clandestine est émaillée de toutes sortes de petits faits, des actes d'entraide et des frictions. Elle est soutenue par la connivence ou une complicité passive. À part dans de petits groupes d'amis, la solidarité se limite, souvent, à ne pas se désolidariser du groupe, être membre d'un groupe, c'est ne pas fragiliser sa cohésion, parfois quoiqu'il en coûte. Participer à une institution, résister à ses règles, les contourner ou les enfreindre semble, en revanche, assez facile à raconter.

\section{Conclusion}

L'internat scolaire est un établissement tout entier scolaire. C'est manifeste dans le règlement intérieur qui formalise son organisation spatio-temporelle, les normes qui président à la conduite des loisirs et de la vie domestique. S'inscrire dans un internat c'est, incontestable- 
L'Internat Scolaire

ment, accepter une emprise accrue de l'école. Mais, penser l'école isolément, comme une sorte de bulle, de sanctuaire disent certains, est une perspective réductrice qui ne rend pas compte de la complexité du monde moderne, qui ne permet pas de comprendre ce choix conjoint des parents et des enfants. L'accroissement de cette emprise permet de diminuer d'autres emprises, celle de la vie familiale et du quartier. Il faut reconnaître que cette emprise ne fonctionne qu'en pointillé, à un rythme saccadé. C'est une limite contemporaine: les coupures des fins de semaines et des vacances font passer ces élèves d'un cadre à un autre, de façon brusque et répétitive, de l'internat à la famille et à son environnement.

Mais l'internat scolaire, par la proximité intensive des élèves et des professionnels, déstabilise le cadre normatif de l'école et les stratégies professionnelles. Non seulement les interactions sont démultipliées, mais surtout, elles s'articulent à des situations de la vie quotidienne qui en changent la nature. Au-delà des statuts, elles touchent des personnes et mettent en jeu des émotions de toutes sortes. Maîtriser son implication et la puissance de ses affects n'est facile ni pour les élèves, ni pour les professionnels. Ce contexte collectif met à l'épreuve les compétences sociales attendues à l'école, la tolérance et la solidarité, la discrétion tout autant que le respect. L'impossibilité de s'éviter les uns les autres intensifie la nécessité de dominer ses sentiments qu'ils soient positifs ou négatifs, agréables ou pénibles.

Toutefois, ce qui contribue à rendre cette emprise acceptable et cette vie collective pacifique, c'est ce qui déborde ce qui est attendue, d'une part, la reconfiguration des rôles professionnels et la personnalisation des relations, et d'autre part, les contournements, les discordances et les arrangements par rapport aux règles prescrites. Ce n'est pas l'exclusivité de l'internat, mais, paradoxalement, comme le souligne Goffman, l'hébergement donne une ampleur à la programmation de ces établissements qui ouvre des possibles et, simultanément, en affaiblit la rigueur. C'est ainsi que nous avons pu repérer trois types d'arrangements, le traitement au cas par cas, la négociation, l'accord tacite par l'ignorance feinte. Cela constitue autant de stratégies permettant d'éviter des face-à-face conflictuels.

Ce jeu avec les normes peut étonner. L'attention portée à ce jeu est sous-tendu par la conception de la participation développée par Goffman: même volontaire, elle suscite de l'ambivalence et des tensions. Paradoxalement, ce jeu tient à l'ambition programmatique et temporelle de l'institution totale. L'internat est un type d'établissement scolaire marginal qui donne à voir, comme à la loupe, des bricolages, des résistances tout autant que de l'adhésion. Ne faut-il pas voir là une dynamique caractéristique d'un monde démocratique, complexe et incertain, dans lequel les acteurs agissent avec une certaine autonomie?

Reçu le 23 décembre 2015

Approuvé le 11 mai 2016

1000 Educação \& Realidade, Porto Alegre, v. 41, n. 4, p. 985-1002, oct./déc. 2016. 


\section{Notes}

1 Disponible sur:<http://eduscol.education.fr/cid47681/l-internat-dispositif-accompagnement.html>. Access on: 28 sept. 2015.

2 Cette collecte a été conduite dans le cadre d'une recherche financée par l'Agence pour la cohésion sociale et l'égalité des chances (ACSÉ) et pilotée par l'Institut français d'éducation l'(IFÉ) sous la direction de Patrick Rayou et Dominique Glasman.

\section{References}

BEHAGHEL Luc; DE CHAISEMARTIN Clément; CHARPENTIER Axelle; GURGAND Marc. Les effets de l'internat d'excellence de Sourdun sur les élèves bénéficiaires: résultats d'une expérience contrôlée. Rapport pour le Fonds d'expérimentation de la jeunesse, avec le concours de la DEPP, 11 avril 2013. Disponible sur: <http://www.cnrs.fr/inshs/recherche/docs-vie-labos/sourdun-rapport. pdf $>$. Consulté le: 28 sept. 2015.

BOULIN, Audrey. Les Internats d'Excellence: un dispositif au prisme de l'expérience adolescente. 2013. 397 f. Thèse (Doctorat, Sciences de l'Éducation) - Université Paris Descartes, Paris, 2013.

BOURDIEU, Pierre. Choses Dites. Paris: Éditions de Minuit, 1987.

FRANCE. Ministère de l'Éducation Nationale (MEN). Développement de l'internat scolaire public: journée nationale d'étude et de réflexion. Discours de Jack Lang, ministre de l'éducation nationale. Discours de clôture. Mardi 19 décembre 2000. Disponible sur: <http://trf.education.gouv.fr/pub/edutel/ actu/2000/19_12_discours_lang.pdf >. Consulté le: 28 sept. 2015.

FRANCE. Inspection générale de l'Éducation Nationale (IGEN). Inspection générale de l'administration de l'éducation nationale et de la recherche (IGAENR). Internato scolaire public: les leçons du terrain. Rapport à Monsieur le ministre de la jeunesse, de l'éducation nationale et de la recherche, à Monsieur le ministre délégué à l'enseignement scolaire, n. 02-042/02-043, juillet 2002. Disponible sur: <http://media.education.gouv;fr/file/05/4/6054.pdf>. Consulté le: 28 sept. 2015.

GLASMAN, Dominique. Internato Scolaire. Travail, cadre, construction de soi. Rennes: PUR, 2012.

GOFFMAN, Erving. Asiles. Études sur la condition des malades mentaux. Paris: Éditions de Minuit, 1968. (Asylums, New York, Anchor books).

GUIGUE, Michèle. L'émergence des interprétations. Propositions pour une épistémologie des traces. Les Sciences de L'Éducation pour l'Ère Nouvelle, Université de Caen-Basse-Normandie, v. 45, n. 4, p. 59-76, 2012.

GUIGUE, Michèle (Org.). Les Déchirements des Institutions Éducatives. Jeux d'acteurs face au décrochage scolaire. Paris: L'Harmattan, 2013.

GUIGUE, Michèle. Ethnographies de L'École. Bruxelles: De Boeck, 2014.

HUGHES, Everett Cherrington. The Sociological Eye. Selected papers. Chicago: Aldine, 1971. (Le regard sociologique. Paris: EHESS, 1996).

JULIA, Dominique. Les Trois Couleurs du Tableau Noir. La Révolution. Paris: Éditions Belin, 1981.

MAYEUR, Françoise. Histoire de l'Enseignement et de l'Éducation III. Paris: Éditions Perrin, 2004. 1789-1930.

Educação \& Realidade, Porto Alegre, v. 41, n. 4, p. 985-1002, oct./déc. 2016. 
PROST, Antoine. Histoire de l'Enseignement en France 1800-1967. Paris: Armand Colin, 1968.

RAYOU, Patrick; GLASMAN, Dominique (Org.). Les internats d'Excellence: un nouveau défi éducatif? Rapport de recherche réalisé avec le soutien de l'ACSE. Lyon: Centre Alain Savary-IFE-ENS, oct. 2012.

ROGERS, Rebecca. Les Bourgeoises au Pensionnat. l'Éducation féminine au XIXème siècle. Rennes: PUR, 2007.

SALAÜN, Marie. L’Ècole Indigène. Nouvelle-calédonie (1885-1945). Rennes: PUR, 2005.

THIERCÉ, Agnès. Histoire de l'Adolescence (1850-1914). Paris: Belin, 1999.

VIGARELLO, Georges. Le Corps Redressé, Histoire d'un Pouvoir Pédagogique. Paris: Armand Colin, 2004

Michèle Guigue est professeure émérite de l'université Charles de Gaulle Lille 3 (France). Après avoir dirigé l'équipe de recherche PROFÉOR, elle est désormais membre du Centre Interuniversitaire de Recherche en Éducation de Lille (CIREL). Elle travaille, dans une perspective ethnographique, sur la vie sociale et le fonctionnement institutionnel des établissements secondaires, les relations entre les différents membres de la communauté éducative, élèves, professionnels, parents.

E-mail :mguigue@club-internet.fr

Audrey Boulin est docteure en sciences de l'éducation associée aux laboratoires CERLIS (Université Paris Descartes, France) et LIRTES (Université Paris-Est Créteil, France). A la croisée de la sociologie de l'école et de la sociologie de la jeunesse, ses travaux de recherche portent principalement sur l'expérience adolescente dans des établissements scolaires, tels que les internats.

E-mail : audrey.boulin@hotmail.fr 